Fashion

\section{Create your own}

\section{VIP+ T-Shirt!}

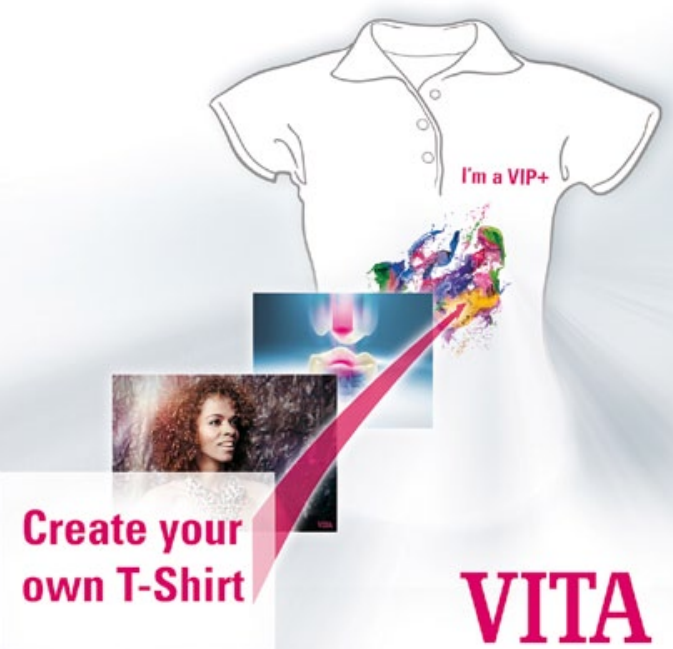

Mit dem neuen VITA T-Shirt-Configurator kann jeder mit nur wenigen Mausklicks sein persönliches VIP+T-Shirt designen und so sein Team mit einem einheitlichen Shirt ausstatten! „I'm a VIP+“ sagt derjenige, der VITAPAN PLUS - die Frontzahnlinie und die weiteren Produkte der VITA Zahnfabrik in sein Herz geschlossen hat. „I'm a VIP+“ gilt für alle, die ihren besonderen Status zum Ausdruck bringen wollen. Mit diesem Slogan legt der neue VITA T-Shirt-Configurator den Grundstein des Designs. Daneben haben die Nutzer freie Hand und können Größe, Kategorie und Anzahl selbst bestimmen. Es kann zwischen Rundhals, V-Ausschnitt und Poloshirt für Damen oder Herren in den Größen S, M, L und XL gewählt werden. Wer es einfach mag, sucht sich ein passendes Motiv aus den über 70 VITA-Vorlagen aus. Alternativ kann das T-Shirt mit einem eigenen Foto, Logo bzw. Bildmotiv selbst gestaltet werden.

Die Vorgehensweise ist hierbei sehr einfach: Bilddatei einfach direkt vom Computer hochladen und virtuell auf der Vorderseite des T-Shirts platzieren. Einmal hochgeladen kann das Motiv individuell angepasst werden. Etwaige Bildrechte sollten nicht verletzt werden und die Vorlage einer Auflösung von min. 300 dpi entsprechen. Einfach Einloggen unter www.vita-zahnfabrik.com/T-ShirtConfigurator und der Kreativität sind keine Grenzen gesetzt!

Nach einer Pressemitteilung der

VITA Zahnfabrik H. Rauter GmbH \& Co. KG,

Bad Säckingen
Implantologie

\section{Implantat für schmale Knochenkämme}

Die neue Mini-Ausführung des einteiligen Locator $^{\circledR}$ Implantats GoDirect ist da. Mit nur $3 \mathrm{~mm}$ Durchmesser ist das Implantat für die lappenlose Implantatinsertion entwickelt worden - insbesondere bei schmalen Knochenkämmen. Es bietet alle Vorteile des Locators in einer einteiligen Konstruktion und ist somit eine kostengünstige Lösung für Hybridbehandlungen. Das System ist in 4 unterschiedlichen Längenoptionen $(10,11,5,13$ und $16 \mathrm{~mm}$ ) und 2 Kragenhöhen (1,5 und

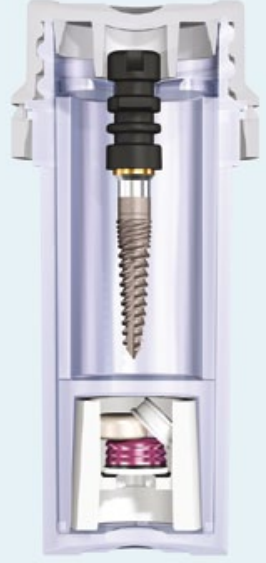

$3 \mathrm{~mm}$ ) erhältlich. Der konische selbstschneidende Implantatkörper trägt zur Primärstabilität bei. Vierfach geführte Mini-Gewinde im Halsbereich verringern Spannungen auf den krestalen Knochen. Das All-in-One GoDirect Mini-Packaging enthält Einbring- und Abdruckpfosten, eine Snapon-Komfortkappe sowie GPSKomponenten. Gratis-Infoline unter 0080040304030.

Nach einer Pressemitteilung der Implant Direct Sybron Europe AG, CH-Zürich Internet: www.implantdirect.de

\section{Auszeichnung}

\section{Medizinischer Luftdekontaminierer mit 2 Goldmedaillen ausgezeichnet}

Für seine innovative und patentierte Luftdekontaminierungstechnologie „DBD-Lyse“ erhielt das ProtectAir-Gerät von Beewair unlängst gleich 2 Goldmedaillen. Die des begehrten ConcoursLepine-Wettbewerbs für herausragende Innovationen technischer Art und die Sonderauszeichnung des französischen Außenhandelsministeriums. Beide Preise gab es aus Frankreich, denn dort sind die gesetzlichen Grundlagen für die hygienische Aufbereitung von Innenraumluft seit dem „Jahr der Luft“ 2013 bereits deutlich gestiegen.

Mit den innovativen ProtectAir-Geräten ist es möglich, Viren, Bakterien, Sporen, Pilze, flüchtige Verbindungen und Allergene aus der Raumluft zu eliminieren und diese in ihren ursprünglichen - reinen - Zustand zurückzuführen. Ideal für die Schaffung von Reinlufträumen in chirurgischen Umgebungen, Behandlungszimmern aber auch Wartezimmern. Hier schützt das Gerät Patienten und das Praxisteam vor Viren und Bakterien, die sonst zu einer möglichen Kreuzkontamination führen könnten. Gleiches gilt für nosokomiale Infektionen in Kliniken. Genauso lässt sich das Gerät aber auch in Privathaushalten einsetzen, um die Widerstandskräfte der dort lebenden Personen zu unterstützen und um für mehr
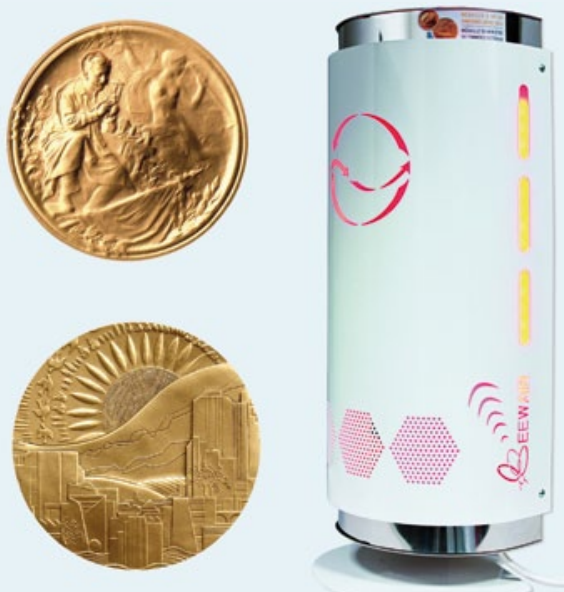

Vitalität zu sorgen. Dank der DBD-Lyse werden auch flüchtige Verbindungen (Lösungsmittel aus Farben und Klebern, Rückstände chemischer Desinfektionmittel etc.) aufgespalten, sodass diese der Körper nicht mehr aufnehmen kann und zu einer Reduzierung des allergenen Potenzials beiträgt. „Das ProtectAir von Zantomed schafft eine reinere Raumluft und unterstützt so das Immunsystem und die Gesunderhaltung aller im Raum befindlicher Personen“, so Benjamin Hatzmann, Geschäftsführer des Anbieters medizinischer Innovationsprodukte.

Nach einer Pressemitteilung der Zantomed GmbH, Duisburg Internet: www.zantomed.de 
Adhäsivtechnik

\section{Befestigungszement}

überzeugt im

\section{Langzeiteinsatz!}

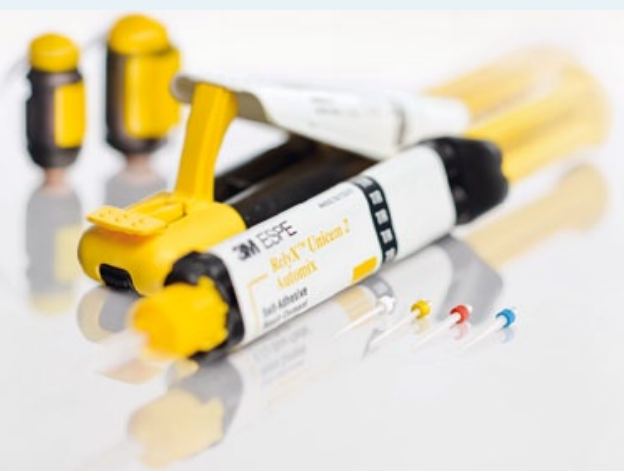

Es ist schon mehr als 10 Jahre her, dass 3M ESPE mit RelyX Unicem den 1. selbstadhäsiven Komposit-Befestigungszement einführte. Seitdem steht das Produkt für die Kombination aus zuverlässigen klinischen Eigenschaften und einer einfachen Anwendung. Der Zement eignet sich für die definitive Eingliederung von Inlays, Onlays, Kronen und Brücken aus Vollkeramik, Komposit oder Metall sowie für die Zementierung von Wurzelkanalstiften und Restaurationen auf Implantatabutments. Das dualhärtende Befestigungsmaterial wurde zunächst in Kapseln und nachfolgend im beliebten Clicker Dispenser eingeführt. Schließlich stellte 3M ESPE eine weiterentwickelte Variante auch in einer Automix-Spritze unter dem Namen RelyX Unicem 2 Automix zur Verfügung. Ganz unabhängig von der Wahl der Darreichungsform ermöglicht das Produkt einen vereinfachten Befestigungsprozess, da das Material selbstadhäsiv und feuchtigkeitstolerant ist. Durch diese Eigenschaften entfällt die Notwendigkeit, den Zahn durch Ätzen, Primen und Bonden zu konditionieren. Gleichzeitig wird eine zuverlässige Haftung sichergestellt.

Dank einer neuen Auszeichnung der unabhängigen Publikation THE DENTAL ADVISOR (www.dentaladvisor.com) besteht erneut ein Anlass, die Erfolgsgeschichte des Materials zu feiern: RelyX Unicem wurde 10 Jahre im klinischen Einsatz getestet und erhielt für seine Leistungsfähigkeit in diesem Langzeittest die Höchstbewertung von 5 Pluszeichen.

Nach einer Pressemitteilung der

3M Deutschland AG, Seefeld

\section{Prophylaxe \\ Hochwertige Ultraschallspitzen}

Die Ultraschallspitzen der neuen PiezoLine von Komet erleichtern das Instrumentieren bei einem Prophylaxe- bzw. PA-Recalltermin. Die effektive Abtragsleistung wird dank piezoelektrischem Handstück (von EMS oder Tekne Dental) durch die 2-seitig lineare Arbeitsweise erreicht. Die PiezoLine für Prophylaxe umfasst 5 Scaler. Das Bakterienmanagement in der Tiefe für parodontal-prophylaktische Anwendungen wird durch weitere 5 Ultraschallspitzen abgedeckt. Für die professionelle Implantatprophylaxe dient der Einweg Polymer-Pin SF1982 in Kombination mit dem wiederaufbereitbaren Spitzenhalter 1981. Implantat-Halspartien bleiben dadurch glatt, es kommt zu keinen Abrasionen. Der mitgelieferte Drehmomentschlüssel
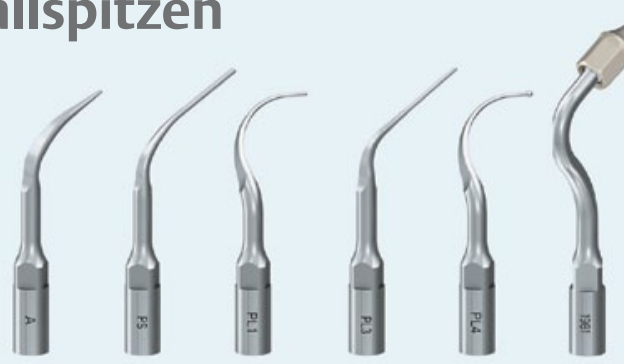

erleichtert die Montage/Demontage der PiezoLine-Ultraschallspitzen, eine Steribox sichert die Aufbewahrung und ein Spüladapter ermöglicht die validierte Aufbereitung in Miele Reinigungs- und Desinfektionsgeräten.

Nach einer Pressemitteilung der

Komet Dental Gebr. Brasseler GmbH \& Co KG, Lemgo

Internet: www.kometdental.de

\section{Minimalinvasive Therapie \\ Patentierte Glasfaserverstärkungen für die tägliche Zahnheilkunde}

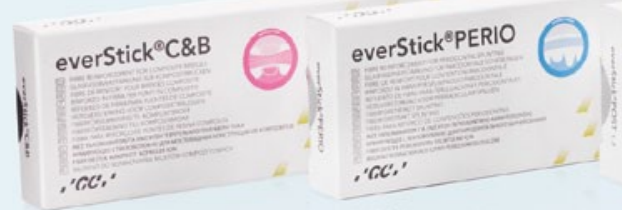

GC hat mit der everStick-Produktpalette leistungsstarke und patientenfreundliche Lösungen für die Zahnheilkunde entwickelt. Das Herz der zugrunde liegenden Faserverstärkung bildet eine patentierte Technologie, die unter anderem vollständige prothetische Therapiemöglichkeiten ermöglicht - minimalinvasiv, ästhetisch und kosteneffizient. Was das gesamte Produktportfolio auszeichnet: Eine Elastizität ähnlich der von Dentin, und hervorragende mechanische Eigenschaften bei der Haftung - basierend auf der patentierten IPN-Struktur (Interpenetrierendes Polymernetzwerk). Auswahl aus dem Produktportofolio: everStickC\&B bietet eine Behandlungsmethode für fehlende Zähne und die Herstellung von glasfaserverstärkten Kompositbrücken innerhalb nur 1 Sitzung. Das breite Indikationsspektrum reicht vom Ersatz fehlender Zähne bis hin zur Erstellung jeglicher Arten an kurz-, mittel- und langfristiger Brücken. Die vorimprägnierte Glasfaserschiene everStickNET ist mit seinem dünnen und bidirekti-

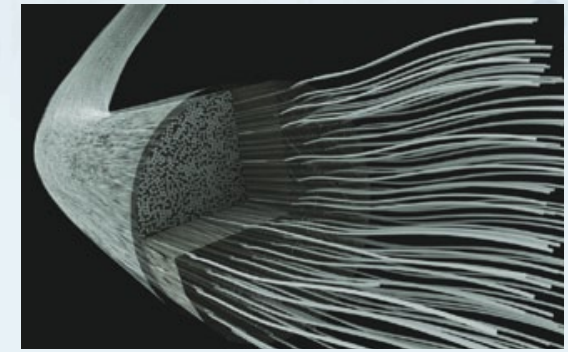

onalen Fasergewebe die Wahl für labiales Splinten bei Verletzungen wie traumatisierten Zähnen oder der Reparatur und Verstärkung von Veneers. Als Glasfaserverstärkung für parodontales Splinten steht everStickPERIO zur Verfügung. Für komplizierte Stift-, Stumpf- und Wurzelkanalrestaurationen bietet sich everStickPOST an. Die Glasfaserverstärkung everStickORTHO ist für die ästhetische Retentionsphase nach der aktiven kieferorthopädischen Behandlung vorgesehen.

Nach einer Pressemitteilung der

GC Europe N.V., B - Leuven

Internet: www.gceurope.com 


\section{Vorhersagbare Remodellierung und Regeneration}

Zimmer Dental erweitert sein umfangreiches regeneratives Produktsortiment um eine weitere Option und gibt die Markteinführung der CopiOs ${ }^{\circledR}$ Xenograft SpongiosaPartikel in Deutschland bekannt. Das Produkt besteht aus mineralisierten, spongiösen Knochenpartikeln boviner Herkunft. Sie werden mit dem proprietären Tutoplast-Prozess hergestellt, bei dem die wertvolle Kollagenmatrix konserviert wird und die osteokonduktiven Eigenschaften erhalten bleiben. Die Besonderheit: Aufgrund ihrer Eigenschaften und Zusammensetzung ermöglichen die Partikel einen Umbau in

Tudor C et al. Oral Surg Oral Med Oral Pathol Oral Radiol Endod. 2008; 105: 430-436

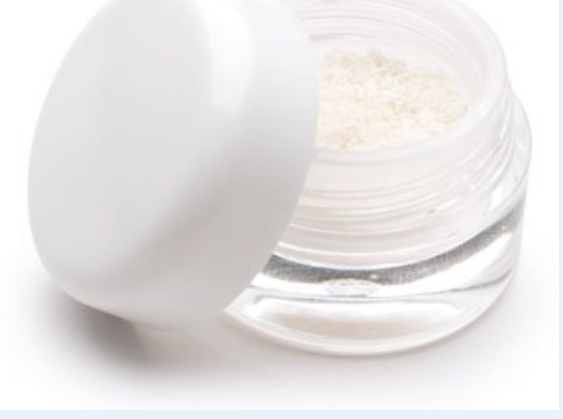

vitalen Knochen ${ }^{1}$. Das Material wird in der Orthopädie bereits seit Jahren erfolgreich eingesetzt.

Nach einer Pressemitteilung der

Zimmer Dental GmbH, Freiburg

Internet: www.zimmerdental.de

\section{Für patientenindividuelle Implantatversorgung}

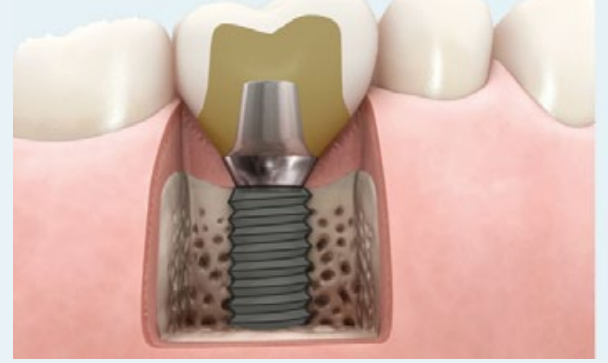

Mit dem ATLANTIS-System entwirft und fertigt DENTSPLY Implants nach einfacher Übermittlung digitaler Laborscans spezifische, passgenaue Abutments und Suprastrukturen. Die CAD/CAM-Restaurationen gleichen dem Original in Größe und Form, passen exakt zur Geometrie des Patientenmundes und können mit allen verfügbaren Implantatsystemen kombiniert werden. Qualität, Preis sowie eine zügige Lieferung sparen sowohl Zeit als auch Ressourcen.

Die Abutments sind variabel in der Kombination sowohl mit zementierten als auch mit verschraubten Lösungen. Ausgehend von der idealen Formgebung der Krone werden mithilfe der patentierten VAD (Virtual Abutment Design)-Software patientenindividuelle CAD/ CAM-Abutments entworfen. Im Vergleich zu Standardabutments werden bei Konzeption und Herstellung der Abutments biologische, anatomische und mechanische Parameter

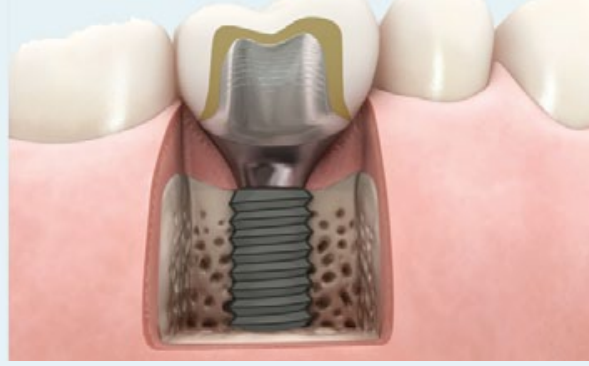

der spezifischen Zahnlücke, des umgebenden Weichgewebes sowie der Nachbarzähne des jeweiligen Patienten berücksichtigt. Neben den Abutments bietet das ATLANTIS-System mit ISUS ein umfassendes Sortiment an Suprastrukturen für festsitzenden und herausnehmbaren implantatgestützten Zahnersatz. Hierdurch ergeben sich zahlreiche Möglichkeiten bei der Entwicklung patientenindividueller Lösungen zur Versorgung von teilund unbezahnten Kiefern. Wie auch bei den ATLANTIS-Abutments wird ausgehend von einem übermittelten diagnostischen Datensatz die benötigte spezifische Suprastruktur mithilfe von CAD/CAM-Technologien entworfen und gefertigt, wobei die Konstruktion mit allen verfügbaren Implantatsystemen kompatibel ist.

Nach einer Pressemitteilung der DENTSPLY IH GmbH, Mannheim

Internet: www.dentsplyimplants.de
Lokalanästhesie

Zahnärzte in vielen Ländern vertrauen darauf

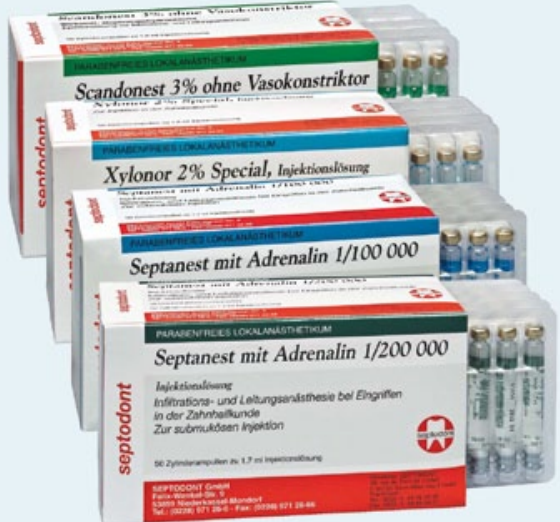

Septodont ist weltweit mit einer Produktion von 500 Millionen Zylinderampullen jährlich einer der führenden Anbieter in der zahnärztlichen Lokalanästhesie. Alleine mit Septanest und dem bewährten Wirkstoff Articain werden jede Sekunde 4 Injektionen rund um den Globus verabreicht. Es ist in 70 Ländern durch die jeweiligen Gesundheitsbehörden zugelassen, insbesondere auch durch die als besonders kritisch geltende amerikanische Zulassungsbehörde FDA.

Die Septanest-Herstellung schließt eine terminale Sterilisation der Zylinderampulle ein. Dieser Sterilisationsprozess erfüllt die höchsten Sterilitätsanforderungen und entspricht dem Goldstandard der Gesundheitsbehörden. Zusätzlich zu den übrigen 27 dokumentierten Qualitätskontrollen wird jede einzelne Zylinderampulle vor der Freigabe nochmals visuell überprüft. Das Produkt ist 100\% latexfrei und kommt während des gesamten Herstellungsprozesses nie mit Latex in Berührung. Zahnärzte und Patienten rund um den Globus profitieren somit von qualitativ hochwertigen und zuverlässigen Produkten. Zusätzlich bietet Septodont in Deutschland Xylonor 2\% Special mit dem Wirkstoff Lidocain und Scandonest 3\% ohne Vasokonstriktor mit dem Wirkstoff Mepivacain an.

Nach einer Pressemitteilung der Septodont GmbH, Niederkassel Internet: www.septodont.de 


\section{0-jåhriges Jubilåum}

Okklusionsprüfung und -korrektur gehört zu den anspruchvollsten Bereichen der modernen Zahnmedizin. Die Belastung und das Wohlbefinden des Patienten hängen von einer sorgfältig abgestimmten Okklusion ab. Diese Sorgfalt garantieren HANEL-Produkte nun schon seit 50 Jahren. Das Unternehmen bietet ein komplettes Programm für die Okklusionsprüfung in Labor und Praxis, sowie optimale Instrumente und anderes Zubehör für die leichte Handhabung. Jahrzehntelange Erfahrung und Produktoptimierung führen $\mathrm{zu}$ der bekannten Qualität. HANEL-Okklusionsprüfmittel zeichnen zuverlässig auf jedem Material. Selbst unter schwierigen Bedingungen sowie in unterschiedlichen Anwendungsbereichen ist eine punktgenaue Okklusionsdarstellung gewährleistet. Die Prüfmittel haben die erforderlichen Stärken, Anschmiegsamkeit, Reißfestigkeit und Farbabgabe, sodass punktförmige, flächige oder strichförmige Kontakte und Mikrokontakte sicher aufgezeigt werden.
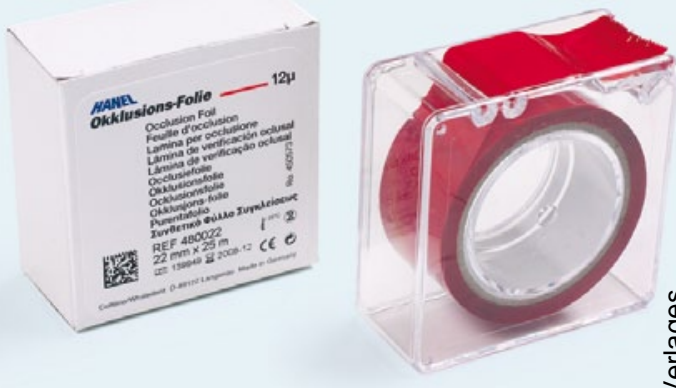

Nach einer Pressemitteilung der Coltène/Whaledent $\mathbf{G m b H}+\mathbf{C o}$. KG, Langenau Internet: www.coltene.com

\section{Für Zahnärzte und Dentalhygienikerinnen}

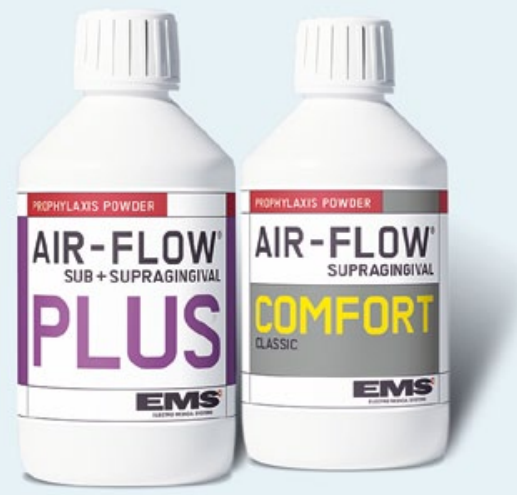

Am 1. März 2014 geht es los: In rund 10 Minuten sind die 21 Online-Fragen rund um das Biofilmmanagement sowie
Air-Flow schnell beantwortet. Fürs Mitmachen schickt EMS der Zahnarztpraxis je eine Flasche Air-Flow Pulver Plus und Air-Flow-Pulver Classic Comfort im Wert von zusammen $47 €$. Die Praxis kann die 2 Pulversorten testen und sich davon ihr eigenes Bild machen. Die Zahl der Teilnehmer ist auf 5000 begrenzt. Daher sollte jede interessierte Zahnarztpraxis www. airflow-dialog.de vor Ende der Kampagne am 30. Juni 2014 besuchen. Über die Fragen hinaus erhält der Besucher eine Menge an Informationen über 30 Jahre Air-Flow, klinische Evidenz, Produkte und die Vision des Prophylaxe-Spezialisten.
Auf der IDS 2013 präsentierte das Unternehmen zum 1. Mal die ProphylaxePulver der neuesten Generation. Das neue Air-Flow-Pulver Plus eignet sich sowohl für sub- als auch supragingivale Behandlungen. Für besonders hartnäckige Verfärbungen im supragingivalen Bereich empfiehlt EMS das Air-Flow-Pulver Classic Comfort. Dieses ist genauso effizient wie das altbewährte Classic-Pulver, zugleich aber aufgrund seiner kleineren Korngröße viel angenehmer für den Patienten.

Nach einer Pressemitteilung der

EMS Electro Medical Systems GmbH, München Internet: www.ems-dent.com

\section{YouTube-Kanal}

\section{Film ab!}

Ein Bild sagt mehr als 1000 Worte, heißt es. Ein Video setzt Bilder in Bewegung um und vermittelt Informationen kompakt und leicht zugänglich. Deshalb stellt Heraeus Kulzer auf einem eigenen YouTube-Kanal Zahnärzten und Zahntechnikern jetzt Wissenswertes rund um Produkte, Dienstleistungen und Forschungsergebnisse zur Verfügung. In den online verfügbaren Videos sind die Informationen verdichtet und leicht verständlich aufbereitet. Neben praktischen Tipps und Tricks zu den Produkten und ihrer Anwendung liefern sie Hintergrundinformationen zu den Herstellungsverfahren. Interessierte finden auf dem Kanal zum Beispiel Videos zur digitalen Abformung mit dem Intraoralscanner cara TRIOS - anschaulich erklärt an einem echten Patientenfall von der Präparation bis zur Fertigstellung des Zahnersatzes. Wer sich für den YouTube-Kanal registriert, erfährt regelmäßig Neuigkeiten rund um die Produkte, Innovationen und aktuelle Studien. Angemeldete Nutzer können zudem Kommentare und Bewertungen abgeben. Der Kanal von Heraeus Kulzer ist über www.youtube.com/ HeraeusDental zu erreichen.

Nach einer Pressemitteilung der Heraeus Kulzer GmbH, Hanau Internet: www.heraeus-kulzer.com

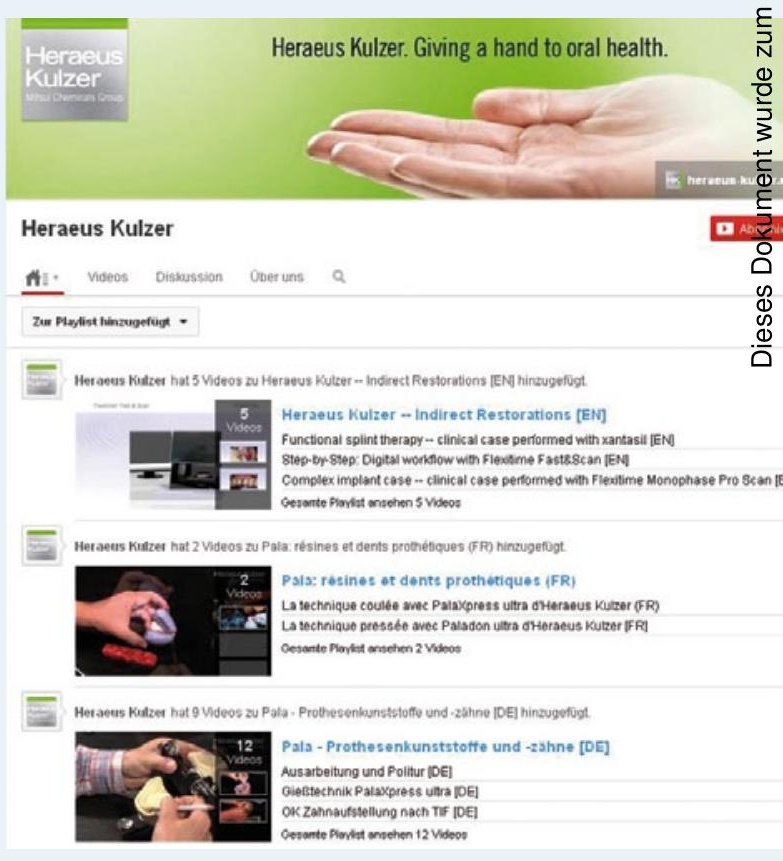




\section{Schmerzlindernder Spezialsitz}
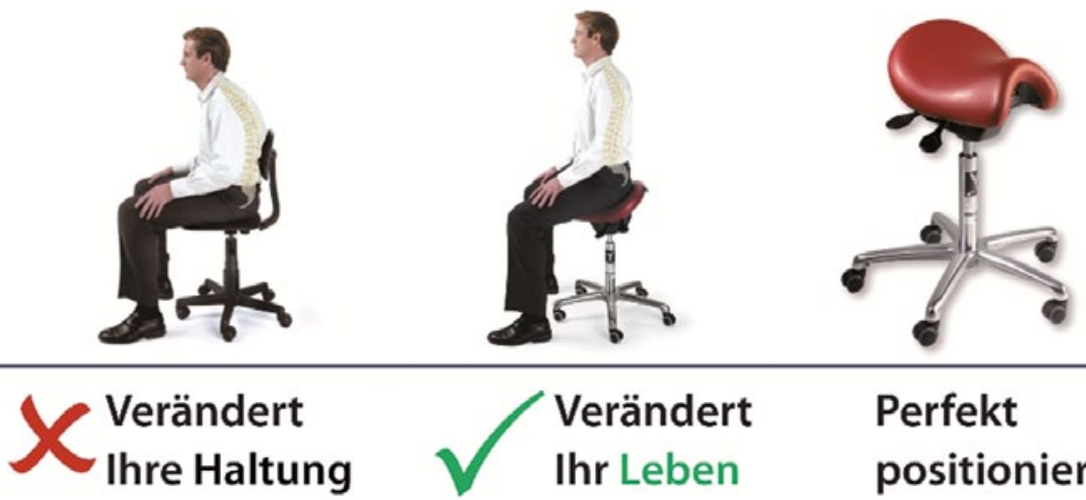

Verändert

Perfekt

Ihr Leben positioniert

Der beliebte Bambach Sattelsitz von Hager \& Werken ist ein ergotherapeutischer Spezialsitz für den Einsatz am Behandlungsstuhl oder im Labor. Der patentierte Sattelsitz zeichnet sich durch seine Sitzfläche aus, die beim Sitzen ganz automatisch den natürlichen S-förmigen Verlauf der Wirbelsäule herstellt. Das Ergebnis ist eine ausgeglichene, rückenfreundliche Sitzhaltung, die länger fit hält und hilft, Rückenschmerzen zu vermeiden. Der Anwender erhält beim Sitzen eine natürliche Becken- und Wirbelsäulenposition aufrecht und mindert dadurch signifikant den Bandscheibendruck und reduziert Spannungsschmerzen. Neben der Höhe lässt sich der Neigungswinkel der Sitzfläche anpassen. Das kommt der typischen, nach vorne geneigten Arbeitshaltung des Behandlers bzw. Zahntechnikers entgegen. Weitere Informationen zum Bambach Sattelsitz erhalten Sie bei Hager \& Werken, wie auch die Möglichkeit einen kostenlosen Praxistest zu vereinbaren.

Nach einer Pressemitteilung der Hager \& Werken GmbH \& Co. KG, Duisburg Internet: www.hagerwerken.de

\section{Stiftung Warentest}

\section{Elektrische Zahnbürsten}

Schon rund einen Monat vor dem Weihnachtsfest gab es im November 2013 für Oral-B Anlass zum Feiern. Der Grund: das Testergebnis der Stiftung Warentest. Die Verbraucherorganisation hatte erneut elektrische Zahnbürsten unter die Lupe genommen, wobei sich die Oral-B Professional Care 1000 an die Spitze der im Testlauf 12/2013 getesteten Modelle setzen konnte. Welche Bedeutung dieses Ergebnis für die Praxis hat, erklärt Jens Starke-Wuschko, Country Manager Professional Oral Health Deutschland/Österreich/ Schweiz bei Procter \& Gamble: „Insbesondere in der wichtigsten Kategorie, der Zahnreinigung, konnte die Oral-B Professional Care 1000 überzeugen. Denn mit der Note 1,3 schnitt sie nicht einfach nur sehr gut, sondern von allen aktuell getesteten AkkuZahnbürsten am besten ab. Zusammen mit ihrer guten Note für die Handhabung $(2,4)$ und dem ,sehr gut' $(1,0)$ in puncto Haltbarkeit und Umwelteigenschaften ergibt sich das überzeugende Gesamturteil.“

Viele Patienten sprechen Testergebnisse wie dieses bei ihrem nächsten Zahnarztbesuch an. „Für eine fundierte Empfehlung lohnt es sich deshalb, über den Ausgang solcher Warentests informiert zu sein." so StarkeWuschko.

Nach einer Pressemitteilung der Procter \& Gamble Germany GmbH, Schwalbach am Taunus

Internet: www.dentalcare.com 